\title{
A Dose-Response Study to Assess the Effects of New Zealand Pine Bark Extract on Glycaemic Responses in Healthy Participants ${ }^{+}$
}

\author{
Wen Xin Janice Lim ${ }^{1}$, Pamela von Hurst ${ }^{2}$, Cheryl Gammon ${ }^{1}$, Lynne Chepulis ${ }^{3}$ \\ and Rachel Page $4,5, *$ \\ 1 School of Health Sciences, Massey University, Auckland 0632, New Zealand; \\ W.X.J.Lim@massey.ac.nz (W.X.J.L.); C.Gammon@massey.ac.nz (C.G.) \\ 2 School of Sport, Exercise and Nutrition, Massey University, Auckland 0632, New Zealand; \\ P.R.vonHurst@massey.ac.nz \\ 3 Waikato Medical Research Centre, University of Waikato, Hamilton 3216, New Zealand; \\ lynnec@waikato.ac.nz \\ 4 School of Health Sciences, Massey University, Wellington 6021, New Zealand \\ 5 Centre for Metabolic Health Research, Massey University, Auckland 0632, New Zealand \\ * Correspondence: R.A.Page@massey.ac.nz; Tel.: +64-4-801-5799 (ext. 63462) \\ † Presented at the 2018 Nutrition Society of New Zealand Annual Conference, Auckland, New Zealand, \\ 28-30 November 2018.
}

Published: 13 March 2019

Background: The current estimation of 451 million people diagnosed with diabetes is expected to increase to 693 million by 2045. Plant extracts have been shown to improve glycaemic control in humans. However, evidence is lacking regarding the hypoglycaemic effects of New Zealand pine bark obtained from Pinus radiata trees.

Methods: An acute, placebo-controlled, single-blind, dose-response, crossover study was designed to investigate the hypoglycaemic potential of increasing doses of New Zealand pine bark extract (supplied by ENZO Nutraceuticals New Zealand Ltd.) on postprandial blood glucose response in healthy individuals.. At each visit, participants arrived fasted for at least $10 \mathrm{~h}$ and were given a single capsule containing either pine bark extract in one of the doses $(50,100 \mathrm{or} 400 \mathrm{mg}$ ) or a placebo (microcrystalline cellulose) to swallow with $150 \mathrm{~mL}$ of water. This was followed by a 2-h oral glucose tolerance test (OGTT). Capillary blood samples were taken at -20, 0, 15, 30, 45, 60, 90 and 120 min. Incremental area under curve (iAUC) of postprandial glucose response during the OGTT was calculated using the trapezoidal rule. Statistical analysis was carried out using a one-way factorial repeated-measures ANOVA (95\% confidence interval) with Bonferroni post hoc analyses.

Results: Eighteen healthy participants (11 women, 7 males, $24.2 \pm 1.0$ years old) completed the study. The mean iAUC of control and pine bark doses of 50, 100 and $400 \mathrm{mg}$ were 318.3 $\pm 26.4,259.5$ $\pm 21.0,285.7 \pm 29.6$, and $242.2 \pm 17.5(\mathrm{mmol} / \mathrm{L} \cdot \mathrm{h})$, respectively, $F(3.00,51.00)=2.90, p=0.049$ across the four intervention arms. Results showed that there was a mean reduction in all doses compared to control of $18.5 \%, 10.2 \%$ and $23.9 \%$, respectively. Although no significant dose response relationship was detected, post hoc test showed a significant iAUC reduction of $76.1 \pm 25.1(\mathrm{mmol} / \mathrm{L} \times \mathrm{h})$ at 400 mg compared to control, $p=0.046$.

Conclusions: Results from the present study suggest that New Zealand pine bark may have hypoglycaemic effects in healthy participants and could potentially be beneficial for individuals with impaired glycaemic control. 
Supplementary Material: The poster is available online at www.mdpi.com/504-3900/8/1/52/s1. 